\title{
Frequency-Dependent Inversion of Net Striatal Output by Endocannabinoid-Dependent Plasticity at Different Synaptic Inputs
}

\author{
Louise Adermark ${ }^{1,2}$ and David M Lovinger ${ }^{1}$ \\ ${ }^{1}$ Section on Synaptic Pharmacology, Laboratory for Integrative Neuroscience, National Institute on Alcohol Abuse and Alcoholism/National Institutes of \\ Health, Bethesda, Maryland 20892, and 2Addiction Biology Unit, Department of Psychiatry and Neurochemistry, Institute of Neuroscience and Physiology, \\ University of Gothenburg, 40530 Gothenburg, Sweden
}

\begin{abstract}
Understanding how striatal neurons integrate glutamatergic and GABAergic inputs is essential for understanding the control of movement and the formation of striatal-based memories. Here we show that GABAergic synapses on striatal medium spiny neurons (MSNs) are more sensitive than glutamatergic synapses on the same cells to endocannabinoid $(\mathrm{eCB})$ signaling, and that protocols that induce short-lasting cannabinoid 1 receptor $\left(\mathrm{CB}_{1} \mathrm{R}\right)$-dependent depression at glutamatergic synapses are sufficient to induce long-term depression (LTD) at GABAergic synapses. We also show that the frequency and duration of glutamatergic input are strong determinants of the net effect of eCB signaling, and key factors in determining if LTD has a net disinhibitory or inhibitory action in striatum. Plastic changes in net output from striatal MSNs are thus a complex function of disinhibitory and inhibitory LTD combined with other forms of synaptic plasticity such as long-term potentiation at excitatory synapses.
\end{abstract}

Key words: anandamide; basal ganglia; LTD; synaptic plasticity; GABA; glutamate

\section{Introduction}

The striatum is the largest nucleus of the basal ganglia and has important roles in planning and execution of controlled movements (Graybiel et al., 1994; Balleine et al., 2007). The majority of synapses $(\sim 80 \%)$ are asymmetric glutamatergic synapses originating from the cortex (associative areas of the frontal and parietal lobes and motor and somatosensory cortex) and thalamus (lateral parafascicular nucleus and the central lateral nucleus) (Wilson, 2007), while the majority of neurons in the striatum (>90\%) are GABAergic medium spiny neurons (MSNs) (Tepper et al., 2007).

The endocannabinoids (eCBs) are key neuromodulatory agents involved in basal ganglia function (eCBs) (Hohmann and Herkenham, 2000; Hilário et al., 2007). eCBs are released postsynaptically and activate presynaptic cannabinoid 1 receptors $\left(\mathrm{CB}_{1} \mathrm{R}\right)$ that depress transmission at both glutamatergic and GABAergic synapses in the basal ganglia (Gerdeman and Lovinger, 2001; Freiman et al., 2006; Narushima et al., 2006; Adermark and Lovinger, 2007a; Lovinger, 2007). The density of $\mathrm{CB}_{1} \mathrm{Rs}$

Received Aug. 12, 2008; revised Dec. 3, 2008; accepted Dec. 29, 2008.

This work was supported by the Division of Intramural Clinical and Basic Research, National Institute on Alcohol Abuse and Alcoholism, National Institutes of Health, The Swedish Society for Medical Research, Swedish Medical Research Council (Diary numbers 2006-2425, 2006-4988, and 2006-6385), Ake Wiberg foundation (113300049), and Swedish Society of Medicine (2008-21390). We are thankful for the help provided by Professor Holger Wigstrom.

Correspondence should be addressed to David M. Lovinger, Section on Synaptic Pharmacology, Laboratory for Integrative Neuroscience, National Institute on Alcohol Abuse and Alcoholism/National Institutes of Health, 5625 Fishers Lane, TS-13, Bethesda, MD 20892. E-mail: lovindav@mail.nih.gov.

DOI:10.1523/JNEUROSCI.3842-08.2009

Copyright $\odot 2009$ Society for Neuroscience $\quad$ 0270-6474/09/291375-06\$15.00/0 is higher at GABAergic than at glutamatergic synapses in striatum (Uchigashima et al., 2007), and our previous research has indicated that $\mathrm{eCB}$ signaling is more easily activated at GABAergic synapses (Adermark and Lovinger, 2007b). Understanding how striatal neurons integrate information from their various synaptic inputs is essential for understanding the control of movement and the formation of striatal-based memories, and the goal of this study was to further evaluate the requirements for eCB signaling and induction of long-term depression (LTD) at glutamatergic and GABAergic synapses in the striatum.

\section{Materials and Methods}

Brain slice preparation. Coronal brain slices containing the striatum were prepared from p16-20 rats as previously described (Adermark and Lovinger, 2007a). Slices were allowed to equilibrate for at least $1 \mathrm{~h}$ in artificial CSF (aCSF) containing (in mM): $124 \mathrm{NaCl}, 4.5 \mathrm{KCl}, 2 \mathrm{CaCl}_{2}, 1 \mathrm{MgCl}_{2}, 26$ $\mathrm{NaHCO}_{3}, 1.2 \mathrm{NaH}_{2} \mathrm{PO}_{4}$, and 10 D-glucose, continuously bubbled with a mixture of $95 \% \mathrm{O}_{2} / 5 \% \mathrm{CO}_{2}$ gas.

Whole-cell voltage clamp recordings. Electrophysiological recordings were performed as previously described (Adermark and Lovinger, 2007a). Internal solutions consisted of (in mM): $120 \mathrm{CsMeSO}_{3}, 5 \mathrm{NaCl}$, 10 TEA-Cl, 10 HEPES, 5 QX-314, 1.1 EGTA, 4 Mg-ATP, 0.3 Na-GTP, for experiments examining EPSCs, and $150 \mathrm{CsCl}, 10 \mathrm{HEPES}, 2 \mathrm{MgCl}_{2}, 0.3$ Na-GTP, $3 \mathrm{Mg}$-ATP, 0.2 BAPTA, for experiments examining IPSCs. For IPSC measurements, $5 \mu \mathrm{M}$ 1,2,3,4-tetrahydro-6-nitro-2,3-dioxobenzo[f] quinoxaline-7-sulfonamide disodium salt (NBQX) and $50 \mu \mathrm{M} 5$ D,L-2-amino-5 phosphonovaleric acid (AP-5) was added to the aCSF. For EPSC recordings AP-5 and $50 \mu \mathrm{m}$ picrotoxin were added to the aCSF. Currents were measured in conventional ruptured-patch whole-cell mode in medium spiny neurons (MSNs) voltage clamped at $-50 \mathrm{mV}$, unless stated. In a subset of recordings MSNs were loaded with the eCB 

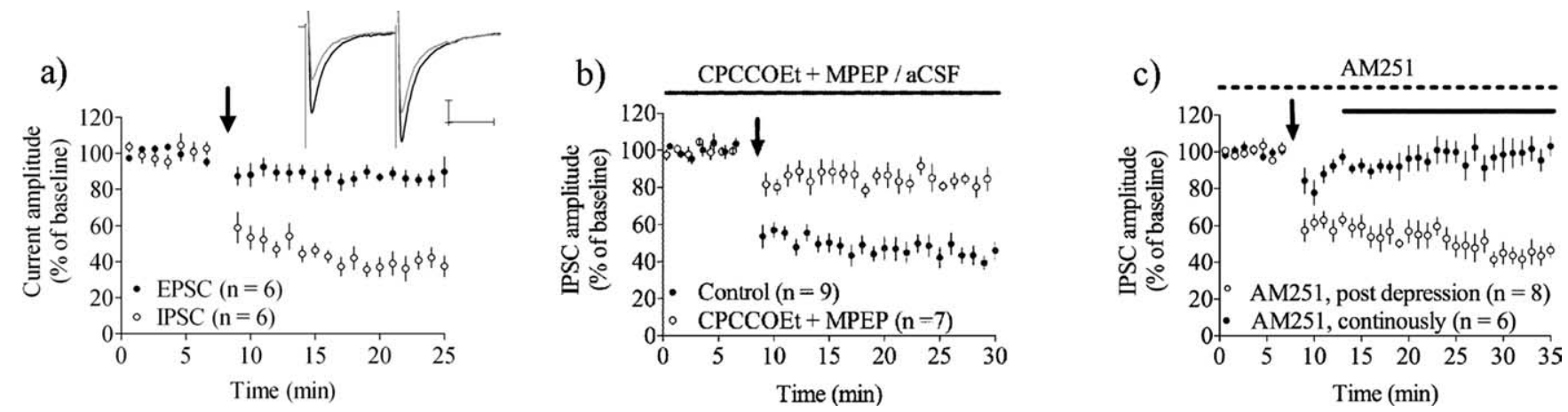

Figure 1. $1 \mathrm{~Hz}$ stimulation for 1 min induces mGluR-dependent LTD at GABAergic synapses. $\boldsymbol{a}$, A train of 60 pulses delivered at $1 \mathrm{~Hz}$ induced LTD at GABAergic synapses, while EPSC amplitude was only slightly decreased. $\boldsymbol{b}$, Stimulation-induced depression at GABAergic synapses required activation of group I mGluRs. $\boldsymbol{c}$, AM251 perfusion prevented depression induced by $1 \mathrm{~Hz}$ stimulation, but was insufficient to reverse an established depression. Example traces show IPSC amplitude at baseline (black) and after 15-20 min (gray). Arrows mark time points when $1 \mathrm{~Hz}$ stimulation was given. Calibration: 100 pA, 25 ms. EPSC/IPSC amplitude data are mean \pm SEM.

arachidonoyl ethanolamide (anandamide, AEA, $50 \mu \mathrm{M}$ ) (Ronesi et al., 2004; Adermark and Lovinger, 2007b). EPSC/IPSC data from AEAloaded cells are shown starting from the point when a stable baseline response was observed which typically occurred within 3 min after establishing the whole cell configuration (Adermark and Lovinger, 2007b).

Unless otherwise stated, baseline synaptic currents were evoked by paired (50 ms interpulse interval) stimuli delivered every $20 \mathrm{~s}$ through a bipolar electrode placed at the border of the dorsolateral striatum and the overlying white matter. Stimulus parameters were adjusted to elicit baseline EPSC or IPSC amplitudes between 200 and 400 pA. Experiments were performed at $29-32^{\circ} \mathrm{C}$, with the temperature not varying more than $11^{\circ} \mathrm{C}$ throughout a given experiment. The amplitude of EPSCs and IPSCs was measured using Clampfit 9.2 as previously described (Adermark and Lovinger, 2007b). Experiments were discontinued if the series resistance varied by $>20 \%$ or increased $>30 \mathrm{M} \Omega$.

Field potential recordings. Extracellular field recordings were conducted as previously described (Yin et al., 2007). Test stimuli were delivered at a frequency of $0.05 \mathrm{~Hz}$ through a bipolar twisted tungsten wire placed at the border of the dorsolateral striatum and the overlying white matter. Slices were perfused at $2.7 \mathrm{ml} / \mathrm{min}$ with regular aCSF, or aCSF containing picrotoxin $(50 \mu \mathrm{M}), \mathrm{AP}-5(50 \mu \mathrm{M})$, or AM251 (3 $\mu \mathrm{M})$. Experiments were performed at $30^{\circ} \mathrm{C}$. The population spike amplitude was measured using Clampfit 9.2, as previously described (Yin et al. 2007).

Statistical analysis. The amplitude of the EPSC, IPSC, or PS, once a stable baseline was achieved, was compared with amplitudes 20-25 min after stabilization of the baseline and presented as mean value $\pm 95 \%$ confidence interval (CI), unless otherwise stated. Clampex 9.2 was used for data acquisition (Molecular Devices), and graphs were assembled in GraphPad Prism (GraphPad Software), and Photoshop. Time course figures are plotted as mean amplitude compared with baseline, with SEM, and two-tailed paired $t$ test was used for statistical analysis.

Drugs. Chemicals were purchased from Sigma or Tocris. AEA was dissolved in ethanol to $25 \mathrm{~mm}$ and used at $50 \mu \mathrm{M}$ in the intracellular solution. NBQX was dissolved in $\mathrm{H}_{2} \mathrm{O}$ to $50 \mathrm{~mm}$ and used at $5 \mu \mathrm{M}$, and $\mathrm{APV}$ and picrotoxin was dissolved in aCSF $(50 \mu \mathrm{M})$. The $\mathrm{CB}_{1}$ receptor blocker AM251 and the $\mathrm{CB}_{1}$ agonist WIN 55,212-2 were dissolved in DMSO to 20 and $10 \mathrm{~mm}$ respectively, stock solutions were diluted in aCSF containing bovine serum albumin (BSA) $(0.5 \mathrm{~g} / \mathrm{L})$ and used at $3 \mu \mathrm{M}$ (AM251) and $1 \mu \mathrm{M}$ or $50 \mathrm{nM}$ (WIN 55,212-2). Cycloheximide was dissolved in aCSF to $80 \mu \mathrm{M}$. Metabotropic glutamate receptor antagonists MPEP and CPCCOEt were dissolved in DMSO to $100 \mathrm{~mm}$ and used at 40 $\mu \mathrm{M}$ (MPEP) and $80 \mu \mathrm{M}$ (CPCCOEt).

\section{Results}

Low frequency stimulus-induced LTD at GABAergic synapses To evaluate eCB signaling and LTD in response to stimulation of intrinsic afferents, we applied a single stimulus train of 60 pulses delivered at $1 \mathrm{~Hz}$ at the border to the white matter overlying striatum. This protocol induced a robust depression of IPSC am- plitude in MSNs voltage clamped at $-50 \mathrm{mV}$ (IPSC amplitude $=$ $39 \pm 11 \%$ of baseline, $n=6, p<0.001$ ), while, as expected, only a small depression was seen at glutamatergic synapses (EPSC amplitude $=87 \pm 8.5 \%, n=6, p<0.01$, IPSC vs EPSC $p<0.001$ ) (Fig. 1a), indicating that this protocol might induce long-lasting disinhibition (DLL) of striatal output. Inhibition of group I mGluRs with combined application of CPCCOEt $(80 \mu \mathrm{M})$ and MPEP $(40 \mu \mathrm{M})$ prevented stimulation-induced depression at GABAergic synapses suggesting that glutamate is vital for this form of depression (IPSC amplitude $=87 \pm 8.7 \%$ of baseline, $n=7, p>0.05$ ) (Fig. 1b). Stimulation-induced depression at GABAergic synapses was abolished in slices treated with AM251 throughout the experiment (IPSC amplitude $=98 \pm 10 \%, n=6$, $p>0.05$ ) (Fig. 1c). However, AM251 treatment beginning 5 min after the end of $1 \mathrm{~Hz}$ stimulation and continuing for up to $60 \mathrm{~min}$ was unable to reverse established depression (IPSC amplitude after 40-45 min of AM251 treatment $=41 \pm 5.7 \%, n=5$ ) (Fig. $1 c)$, suggesting that this stimulation protocol induces LTD at GABAergic synapses in the striatum. The depression in IPSC amplitude induced by a train of 60 pulses at $1 \mathrm{~Hz}$ was not significantly different if the stimulating electrode was placed in the dorsolateral striatum itself (IPSC amplitude (local stimulation) $=53 \pm 13 \%, n=5, p<0.01$ : local vs white matter stimulation, $p>0.05)$.

\section{GABAergic synapses are highly sensitive to $C_{1} R$ activation}

Bath application of a low concentration of the $\mathrm{CB}_{1} \mathrm{R}$ agonist WIN $55,212-2(50 \mathrm{nM})$ was unable to significantly affect postsynaptic currents at glutamatergic synapses (EPSC amplitude $=87 \pm 10 \%$ of baseline, $n=6, p>0.05)$. The same treatment induced a robust depression of GABAergic IPSC amplitude (IPSC amplitude $=51 \pm 21 \%$ of baseline, $n=8, p<0.01$ ), suggesting that GABAergic synapses are more sensitive to $\mathrm{CB}_{1} \mathrm{R}$ activation (Fig. $2 a$ ).

Treatment with a higher concentration of the $\mathrm{CB}_{1} \mathrm{R}$ agonist WIN55,212-2 (1 $\mu \mathrm{M})$ induced a robust depression at both glutamatergic and GABAergic synapses (EPSC amplitude at $t=15-20$ $\min =62 \pm 18 \%$ of baseline, $n=5, p<0.01$; IPSC amplitude $=$ $64 \pm 9.2 \%$ of baseline, $n=12, p<0.001$ ), that was reversed by subsequent application of the $\mathrm{CB}_{1} \mathrm{R}$ antagonist AM251 (3 $\left.\mu \mathrm{M}\right)$ within $15 \mathrm{~min}$ of the onset of antagonist treatment at glutamatergic synapes (EPSC amplitude after 15-20 min AM251 treatment $=87 \pm 10 \%$ of baseline, $n=7, p>0.05$ ), while no recovery was detected at GABAergic synapses (IPSC amplitude after 15-20 $\min \mathrm{AM} 251$ treatment $=50 \pm 14 \%$ of baseline $n=12, p<0.001$ ) (Fig. 2b). 

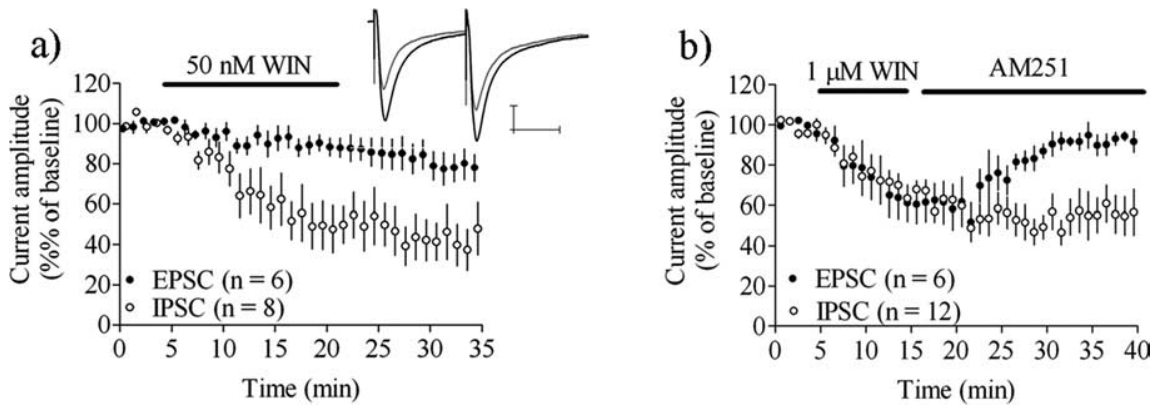

Figure 2. GABAergic synapses are highly sensitive to $C_{1} B_{1}$ Ractivation and LTD induction. $\boldsymbol{a}$, Treatment with a low concentration of the $\mathrm{CB}_{1} \mathrm{R}$ agonist WIN55,212-2 induced a robust depression at GABAergic but not glutamatergic synapses. $\boldsymbol{b}$, Depression in response amplitude produced by WIN55,212-2(1 $\mu \mathrm{M})$ was reversed by AM251 at glutamatergic synapses, but not at GABAergic synapses. Example traces show IPSC amplitude at baseline (black) and after 20-25 min (gray). Calibration: 100 pA, 25 ms. EPSC/IPSC amplitude data are mean \pm SEM.

\section{a)}
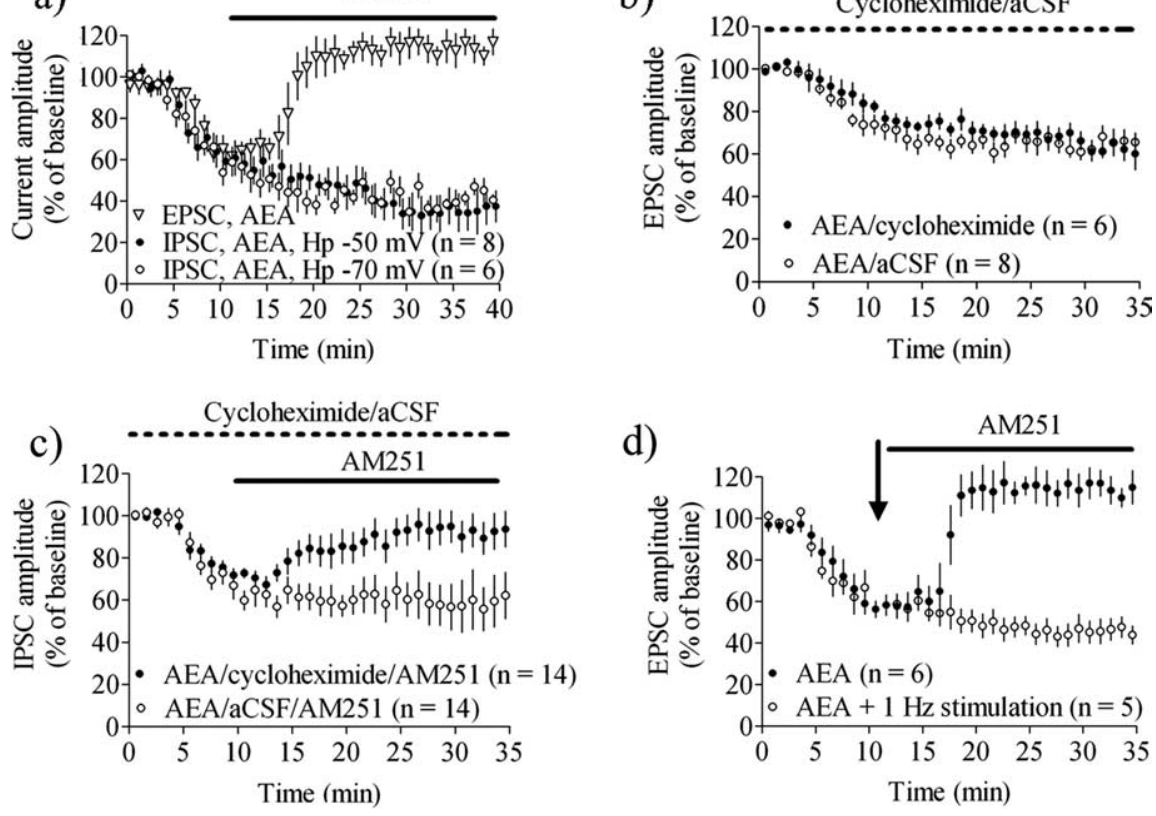

Figure 3. Postsynaptic endocannabinoid loading is sufficient to induce LTD at GABAergic synapses. $\boldsymbol{a}$, AEA-induced depression at GABAergic synapses was not reversed by AM251 even if the postsynaptic cell was held at $-70 \mathrm{mV}$ and IPSCs were activated with single pulse stimulation. $\boldsymbol{b}$, Depression induced by AEA-loading was not inhibited by cycloheximide showing that blockade of protein synthesis does not interfere with eCB release or $\mathrm{CB}_{1} \mathrm{R}$ activation. $C_{\text {, }}$ AEA-induced depression of sIPSCs was reversed by AM251 treatment in cycloheximide-treated slices (filled circles) suggesting that postsynaptic AEA-loading is sufficient to induce LTD at GABAergic synapses, but not glutamatergic synapses. $\boldsymbol{d}$, AEA-induced depression at glutamatergic synapses was reversed by AM251, but when combined with a train of 60 pulses delivered at $1 \mathrm{~Hz}$ the AEA-induced depression was converted to LTD. Arrows mark time point when $1 \mathrm{~Hz}$ stimulation was given. EPSC/IPSC amplitude data are mean values \pm SEM.

AEA loading combined with single pulse stimulation, which is insufficient to induce depression at glutamatergic synapses (Adermark and Lovinger, 2007b), induced depression at GABAergic synapses that was not reversed by subsequent $\mathrm{CB}_{1} \mathrm{R}$ antagonist treatment. This depression was not different in cells clamped at $-50 \mathrm{mV}$ compared with MSNs clamped at $-70 \mathrm{mV}$ (IPSC amplitude $=47 \pm 13 \%$ of baseline, $n=9, p<0.001(\mathrm{Hp}=-50 \mathrm{mV})$, and $40 \pm 8.5 \%$ of baseline, $n=7, p<0.001$ $(\mathrm{Hp}=-70 \mathrm{mV})$ (Fig. 3a).

\section{AEA-Induced LTD at GABAergic synapses is sensitive to protein synthesis inhibition}

The finding that antagonist treatment cannot reverse established synaptic depression suggests that LTD has been induced under many of the experimental conditions mentioned above. To confirm this finding we pretreated slices with the protein translation inhibitor cycloheximide $(80 \mu \mathrm{M})$ that prevents LTD without disrupting $\mathrm{CB}_{1} \mathrm{R}$-dependent short-term depression (Yin et al., 2006). Extracellular perfusion of cycloheximide did not prevent depression of EPSC amplitude induced by postsynaptic AEA, indicating that protein synthesis is not required for $\mathrm{eCB}$ release or $\mathrm{CB}_{1} \mathrm{R}$ activation (EPSC amplitude at $t=20-25 \mathrm{~min}$ in cycloheximide treated slices $=70 \pm 7.5 \%, n=7, p<$ 0.001; EPSC amplitude in control slices $=$ $65 \pm 8.9 \%, n=9, p<0.01$; cycloheximide treated vs aCSF, $p>0.5$ ) (Fig. $3 b$ ). In slices perfused with cycloheximide, AEAinduced depression was reversed by AM251 (IPSC amplitude after 15-20 min AM251 treatment $=94 \pm 14 \%, n=11$, $p>0.65$ ), while no antagonist reversal was observed in the absence of cycloheximide (Fig. 3c). These findings indicate that AEA-loading is sufficient to induce protein synthesis inhibitor-sensitive LTD at GABAergic synapses, which is not the case for glutamatergic synapses.

Previously published data suggest that

To simulate a condition in which eCBs have been produced but are not yet released from the postsynaptic cell we loaded MSNs with the eCB arachidonoylethanolamide (anandamide or AEA) $(50 \mu \mathrm{M})$, which, when combined with paired pulse stimulation induces a robust depression at both excitatory and inhibitory synapses (Ronesi et al., 2004; Adermark and Lovinger, 2007b). Similar to agonist-mediated $\mathrm{CB}_{1} \mathrm{R}$ activation, AEAinduced depression was reversed by subsequent AM251 treatment at glutamatergic synapses, but not at GABAergic synapses (EPSC amplitude at $t=15-20 \mathrm{~min}=64 \pm 5.1 \%$ of baseline, $n=$ 5 , EPSC amplitude at $t=25-30 \mathrm{~min}=114 \pm 9.3 \%$ of baseline, $n=6, p>0.05$; IPSC amplitude at $t=15-20 \mathrm{~min}=62 \pm 11 \%$ of baseline, $n=12, p<0.001$, IPSC amplitude at $t=25-30 \mathrm{~min}=$ $59 \pm 19 \%$ of baseline, $n=12, p<0.001$ ) (Fig. $3 a$ ). Furthermore, induction of eCB-dependent $\mathrm{LTD}$ requires both $\mathrm{CB}_{1} \mathrm{R}$ signaling and another coincident presynaptic signal (Sjöström et al., 2003; Singla et al., 2007; Adermark et al., 2009). It is thus possible that at GABAergic synapses that are rich in $\mathrm{CB}_{1} \mathrm{R}$, LTD may be easily induced even with moderate stimulus trains, while at relatively $\mathrm{CB}_{1} \mathrm{R}$-poor glutamatergic synapses both strong $\mathrm{CB}_{1} \mathrm{R}$ activation and afferent stimulation may be needed to induce LTD. When we combined AEA-loading with one train of 60 pulses delivered at $1 \mathrm{~Hz}$ we observed an inhibition of EPSC amplitude comparable to that observed with AEA loading alone. However, AM251 treatment after combined AEA loading and $60 \mathrm{~s}$, $1 \mathrm{~Hz}$ stimulation did not reverse the depression in EPSC amplitude, indicating that AEA-loading combined with moderate afferent activation converts eCB-induced short-term de- 

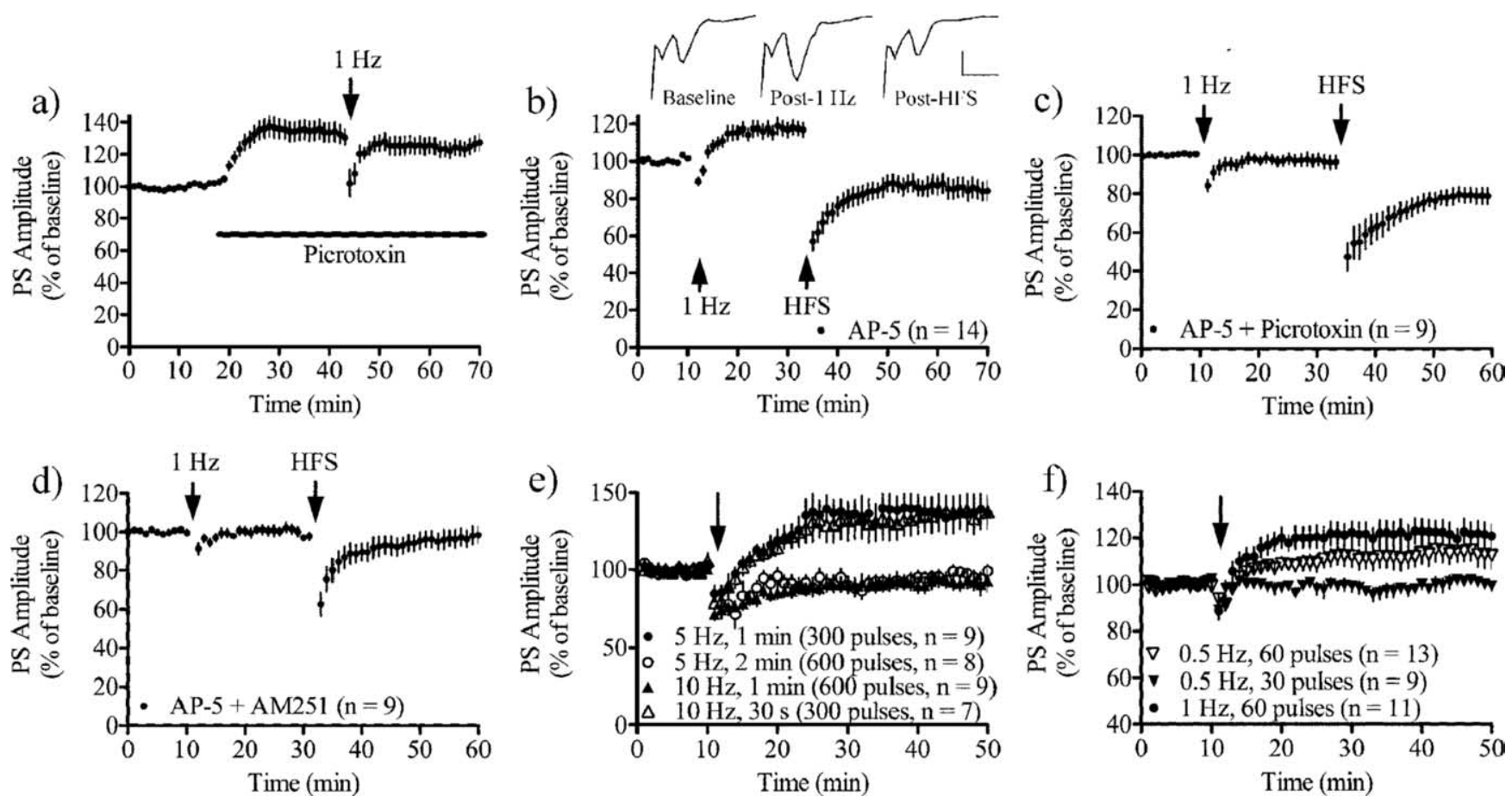

Figure 4. Net output from striatal MSNs is a function of disinhibitory and inhibitory LTD and LTP. $\boldsymbol{a}$, Perfusion of picrotoxin (50 $\mu \mathrm{M})$ increased population spike (PS) amplitude, showing that GABAergic inhibition regulates striatal output. A train of 60 pulses delivered at $1 \mathrm{~Hz}$ did not significantly affect PS amplitude in slices treated with picrotoxin $(n=16)$. $\boldsymbol{b}$, A small but consistent potentiation was detected after $1 \mathrm{~Hz}$ stimulation in AP-5 $(50 \mu \mathrm{m}$ ) treated slices, while HFS ( 4 trains of $1 \mathrm{~s}, 100 \mathrm{~Hz}$ stimulation, intertrain interval $10 \mathrm{~s}$ ) induced LTD. Example traces showing PS at baseline, after $60 \mathrm{~s}, 1 \mathrm{~Hz}$ stimulation and following HFS stimulation in an AP- 5 treated slice. Calibration: $0.2 \mathrm{mV}, 4 \mathrm{~ms}$. c, The $60 \mathrm{~s}, 1 \mathrm{~Hz}$ stimulation protocol did not alter PS amplitude in slices treated with AP-5 and picrotoxin, suggesting that the increase in PS amplitude involves decreased GABAergic inhibition (DLL). $\boldsymbol{d}$, Neither low nor high frequency stimulation affected PS amplitude in slices treated with AP-5 and AM251, suggesting that all plasticity in AP-5-treated slices is mediated through $\mathrm{CB}_{1}$ R activation. $\boldsymbol{e}, 60 \mathrm{~s}, 5 \mathrm{~Hz}$ stimulation induced a robust DLL, while $120 \mathrm{~s}, 5 \mathrm{~Hz}$ had no effect on PS amplitude. In contrast, $60 \mathrm{~s}, 10 \mathrm{~Hz}$ stimulation was insufficient to alter PS amplitude, while $30 \mathrm{~s}, 10 \mathrm{~Hz}$ induced DLL.f, Stimulation for $120 \mathrm{~s}$ at $0.5 \mathrm{~Hz}$ stimulation or $30 \mathrm{~s}$ at $1 \mathrm{~Hz}$, but not $60 \mathrm{~s}$ at $0.5 \mathrm{~Hz}$, induced DLL.

pression to LTD (EPSC amplitude at $t=30-35 \mathrm{~min}=46 \pm$ 9.8\%, $n=5, p<0.001$ ) (Fig. $3 d$ ).

\section{Frequency dependent inversion of striatal output}

Our findings indicate that eCB signaling and LTD are induced at different afferent stimulus frequencies at GABAergic synapses compared with glutamatergic synapses. Thus it is possible that eCB release produced by sustained low frequency synaptic activation produces a long-lasting increase in MSN output, while higher frequency activation produces a net decrease in output in the same set of cells. To test this hypothesis we examined stimulation-induced changes in population spike (PS) amplitude using field potential recording in the dorsolateral striatum. To establish the impact of GABAergic innervation on MSN output, we perfused slices with picrotoxin $(50 \mu \mathrm{M})$, which significantly enhanced PS amplitude (PS amplitude at $t=30-35 \mathrm{~min}=135 \pm$ $11 \%$ of baseline, $n=16, p<0.001$ ) (Fig. $4 a$ ), showing that GABAergic inhibition is important for the modulation of striatal output. PS amplitude was not significantly affected by $60 \mathrm{~s}, 1 \mathrm{~Hz}$ stimulation in slices perfused with picrotoxin $(p>0.05)$ (PS amplitude at $t=55-60 \mathrm{~min}=124 \pm 9.5 \%$ of baseline, $n=16$, $p<0.01$ ) (Fig. 4a).

In normal ACSF, neither $60 \mathrm{~s}, 1 \mathrm{~Hz}$ stimulation nor HFS induced a significant change in PS amplitude (PS amplitude at $t=$ $25-30 \mathrm{~min}=92 \pm 16 \%$ of baseline, $n=12, p>0.05 ; t=45-50$ $\min =119 \pm 20 \%$ of baseline, $n=11, p>0.05)$. It is possible that this result reflects combined activation of long-term potentiation (LTP) (Calabresi et al., 1992), and LTD. We thus examined effects of the two stimulation patterns in slices treated with AP-5, which blocks NMDARs and should prevent LTP. Under this condition, $60 \mathrm{~s}, 1 \mathrm{~Hz}$ stimulation induced a small but consistent increase in PS amplitude (PS amplitude at $t=25-30 \mathrm{~min}=$ $115 \pm 7.3 \%$ of baseline, $n=14, p<0.01)$ indicating that a DLL has occurred, while HFS induced LTD (PS amplitude at $t=$ $45-50 \mathrm{~min}=85 \pm 9.5 \%$ of baseline, $n=13, p<0.01)($ Fig. $4 b)$. PS amplitude was not enhanced by the $60 \mathrm{~s}, 1 \mathrm{~Hz}$ stimulation protocol in slices treated with AP-5 and picrotoxin (PS amplitude at $t=25-30 \mathrm{~min}=97 \pm 6.1 \%$ of baseline, $n=9, p>0.05$; PS amplitude after HFS at $t=45-50 \mathrm{~min}=74 \pm 8.3 \%$ of baseline, $n=9, p<0.001$ ) (Fig. $4 c$ ), and perfusion with AM251 and AP-5 prevented the changes produced by either $60 \mathrm{~s}, 1 \mathrm{~Hz}$ stimulation or HFS (PS amplitude after $1 \mathrm{~Hz}$ stimulation at $t=25-30 \mathrm{~min}=$ $100 \pm 4.0 \%$ of baseline, $n=9, p>0.05$; PS amplitude after HFS at $t=45-50 \mathrm{~min}=92 \pm 9.6 \%$ of baseline, $n=9, p>0.05$ ) (Fig. $4 d$ ). Delivery of HFS before $60 \mathrm{~s}, 1 \mathrm{~Hz}$ stimulation induced a depression in PS amplitude and occluded DLL (PS amplitude at $t=25-30 \mathrm{~min}=76 \pm 12 \%$ of baseline, $n=12, p<0.01 ; t=$ $45-50 \mathrm{~min}=79 \pm 12 \%$ of baseline, $n=9, p<0.05)$.

To determine which stimulus parameters induce DLL, and which produce a switch from DLL to LTD, we applied single stimulus trains delivered at different frequencies and durations. Stimulation with a $60 \mathrm{~s}, 5 \mathrm{~Hz}$ train induced robust DLL (PS amplitude at $t=35-40 \mathrm{~min}=139 \pm 19 \%$ of baseline, $n=9, p<$ 0.01 ), while no change in PS amplitude was seen after $60 \mathrm{~s}, 10 \mathrm{~Hz}$ stimulation (PS amplitude at $t=35-40 \mathrm{~min}=92 \pm 8.6 \%$ of baseline, $n=9, p>0.05 ; 5 \mathrm{~Hz}$ vs $10 \mathrm{~Hz}, p<0.001$ ) (Fig. $4 e$ ). Pretreatment with AP- 5 was not required for $60 \mathrm{~s}, 5 \mathrm{~Hz}$-induced DLL (PS amplitude at $t=35-40 \mathrm{~min}=118 \pm 13 \%$ of baseline, 
$n=8, p<0.05$ ), indicating that DLL can occur under more "natural" conditions. Interestingly, $120 \mathrm{~s}, 5 \mathrm{~Hz}$ stimulation did not affect PS amplitude (PS amplitude at $t=35-40 \mathrm{~min}=93 \pm$ $8.7 \%$ of baseline, $n=9, p>0.05)$, while $30 \mathrm{~s}, 10 \mathrm{~Hz}$ induced DLL (PS amplitude at $t=35-40 \mathrm{~min}=132 \pm 22 \%$ of baseline, $n=9$, $p<0.05$ ) (Fig. 4e), indicating that when afferents are activated at frequencies well within the range exhibited by cortical inputs the experimental outcome is dependent on the duration of the stimulation. We also found that $60 \mathrm{~s}-0.5 \mathrm{~Hz}$ stimulation did not affect PS amplitude (PS amplitude at $t=35-40 \mathrm{~min}=98 \pm 4.3 \%$ of baseline, $n=9, p>0.05$ ), while $120 \mathrm{~s}-0.5 \mathrm{~Hz}$ induced a DLL that was not significantly different in magnitude from DLL induced by $60 \mathrm{~s}, 1 \mathrm{~Hz}$ stimulation (PS amplitude at $t=35-40 \mathrm{~min}=$ $112 \pm 9.4 \%$ of baseline, $n=13, p<0.05 ; 60 \mathrm{~s}, 1 \mathrm{~Hz}$ vs $120 \mathrm{~s}-0.5$ $\mathrm{Hz}, p>0.05$ ) (Fig. $4 f$ ). Our observations suggest that plastic changes in net striatal output involve LTD, LTP and DLL at both GABAergic and glutamatergic synapses, and that the net balance is regulated by the frequency and duration of glutamatergic afferent input.

\section{Discussion}

The data presented here suggest that GABAergic, presumed inhibitory, synapses are more sensitive to eCB signaling and LTD induction compared with glutamatergic excitatory synapses in the striatum. This might involve different presynaptic activity requirements for LTD induction at the two types of synapses (Singla et al., 2007; Adermark et al., 2009), but could also be connected to the high expression of $\mathrm{CB}_{1} \mathrm{R}$ at inhibitory synapses (Uchigashima et al., 2007). AEA-induced depression at GABAergic synapses was reversed by AM251 in the presence of cycloheximide, which provides independent support for the idea that the AEA effect at GABAergic synapses is truly LTD. Our findings also indicate that protocols which induce short-lasting eCB-mediated depression at glutamatergic synapses are sufficient to induce LTD at GABAergic synapses in the dorsolateral striatum.

Interestingly, by pairing postsynaptic eCB-loading with $60 \mathrm{s,} 1$ $\mathrm{Hz}$ stimulation, the short-term depression of glutamatergic transmission normally induced by AEA could be converted to LTD (Fig. 3). Since postsynaptic cells are loaded with $50 \mu \mathrm{M}$ AEA there should be an excess of eCBs at both GABAergic and glutamatergic synapses. Increased stimulation might therefore be required to synergize $\mathrm{CB}_{1} \mathrm{R}$ activation with some presynaptic mechanism for LTD-induction (Sjöström et al., 2003; Singla et al., 2007). It should be noted that WIN55,212-2-induced depression at many synapses, including GABAergic striatonigral synapses, is fully reversed by subsequent AM251 treatment even when afferent activation is given at comparable levels to those used with our single-pulse stimulus protocol (Kreitzer et al., 2002; Wallmichrath and Szabo, 2002). Thus, the conditions necessary to convert $\mathrm{CB}_{1} \mathrm{R}$-mediated short-term synaptic depression to LTD likely vary in a synapse-specific manner.

The necessity for mGluR activation in $1 \mathrm{~Hz}-\mathrm{LTD}$ at GABAergic synapses indicates an important role for glutamatergic inputs in this form of DLL. This finding is in line with data from the hippocampus and basolateral amygdala indicating that glutamatergic synaptic activation of group I mGluRs is critical for afferent stimulus-induced LTD at inhibitory synapses (Chevaleyre and Castillo, 2003; Azad et al., 2004). The role of glutamatergic transmission in this form of LTD indicates that the sustained synaptic depression most likely occurs at GABAergic synapses located on the distal dendrites and dendritic spines of MSNs, since only these synapses would be close to the glutamatergic synapses that are present on the outer two-thirds of the dendritic arbor. These observations suggest the interesting possibility that "feedback" inhibitory inputs from other MSNs are among the GABAergic synapses that exhibit $1 \mathrm{~Hz}$-LTD.

Using different stimulation frequencies $(0.5,1,5,10 \mathrm{~Hz})$ and durations ( $30 \mathrm{~s}, 1 \mathrm{~min}, 2 \mathrm{~min}$ ), we showed that when frequencies within the presumed physiological range are used, the number of pulses used, rather than just the frequency, is what determines whether the stimulation will enhance striatal output through DLL, or whether LTD occurs (Fig. 4e,f). During low frequency, short duration repetitive activation of glutamatergic inputs LTD of GABAergic synapses predominates; giving rise to DLL of striatal output. At higher frequencies, or longer stimulus durations, the glutamatergic synapses themselves are depressed, which would decrease output from projection neurons. The frequency/ duration of glutamatergic input is thus a strong determinant of the net effect of eCB signaling, and a key factor in determining whether LTD has a net disinhibitory or inhibitory action in striatum. Perfusion of picrotoxin amplified PS amplitude showing that GABAergic inhibition regulates striatal output. Furthermore, the increase in PS amplitude in AP-5 treated slices after $60 \mathrm{~s}, 1 \mathrm{~Hz}$ stimulation was blocked by picrotoxin or AM251, which is consistent with the idea that this DLL involves a change in GABAergic transmission mediated through $\mathrm{CB}_{1} \mathrm{R}$ activation. Thus, the striatal MSN shows unique patterns of glutamateactivated eCB-dependent plasticity that might cause frequencydependent inversion of net striatal output.

In vivo, moderate frequency membrane potential oscillations $(5 \mathrm{~Hz}$ ) were recorded in MSNs that were caused by the synchronous firing of cortical inputs (Charpier et al., 1999). In addition, in vitro recordings from layer 5 rat prefrontal cortical neurons demonstrated firing at irregular frequencies with an average of $5-10 \mathrm{~Hz}$ (Fellous et al., 2003). In vivo recordings from the striatum have shown that MSNs fire at frequencies of 1-10 Hz, with occasional bursts reaching at least $30 \mathrm{~Hz}$, while cholinergic neurons fire at $1-3 \mathrm{~Hz}$, and that GABAergic interneurons can fire at $20 \mathrm{~Hz}$ (Burkhardt and Costa, unpublished observations). Since the frequencies used in this study are within the normal physiological firing range of striatal afferents and intrinsic neurons, it thus is likely that DLL is of physiological importance, suggesting that plastic changes in net output from striatal MSNs are a complex function of DLL and LTD combined with other forms of synaptic plasticity such as LTP at excitatory synapses (Calabresi et al., 1992; Partridge et al., 2000). eCB-dependent plasticity at GABAergic and glutamatergic transmission may participate in striatal-based learning and memory by sculpting the net output of individual MSNs.

\section{References}

Adermark L, Lovinger DM (2007a) Combined activation of L-type Ca2 + channels and synaptic transmission is sufficient to induce striatal longterm depression. J Neurosci 27:6781-6787.

Adermark L, Lovinger DM (2007b) Retrograde endocannabinoid signaling at striatal synapses requires a regulated postsynaptic release step. Proc Natl Acad Sci U S A 104:20564-20569.

Adermark L, Talani G, Lovinger DM (2009) Endocannabinoid-dependent plasticity at GABAergic and glutamatergic synapses in the striatum is regulated by synaptic activity. Eur J Neurosci 29:32-41.

Azad SC, Monory K, Marsicano G, Cravatt BF, Lutz B, Zieglgänsberger W, Rammes G (2004) Circuitry for associative plasticity in the amygdala involves endocannabinoid signaling. J Neurosci 24:9953-9961.

Balleine BW, Delgado MR, Hikosaka O (2007) The role of the dorsal striatum in reward and decision-making. J Neurosci 27:8161-8165.

Calabresi P, Pisani A, Mercuri NB, Bernardi G (1992) Long-term Potentiation in the Striatum is Unmasked by Removing the Voltage-dependent 
Magnesium Block of NMDA Receptor Channels. Eur J Neurosci 4:929-935.

Charpier S, Mahon S, Deniau JM (1999) In vivo induction of striatal longterm potentiation by low-frequency stimulation of the cerebral cortex. Neuroscience 91:1209-1222.

Chevaleyre V, Castillo PE (2003) Heterosynaptic LTD of hippocampal GABAergic synapses: a novel role of endocannabinoids in regulating excitability. Neuron 38:461-472.

Fellous JM, Rudolph M, Destexhe A, Sejnowski TJ (2003) Synaptic background noise controls the input/output characteristics of single cells in an in vitro model of in vivo activity. Neuroscience 122:811-829.

Freiman I, Anton A, Monyer H, Urbanski MJ, Szabo B (2006) Analysis of the effects of cannabinoids on identified synaptic connections in the caudate-putamen by paired recordings in transgenic mice. J Physiol 575:789-806

Gerdeman G, Lovinger DM (2001) CB1 cannabinoid receptor inhibits synaptic release of glutamate in rat dorsolateral striatum. J Neurophysiol 85:468-471.

Graybiel AM, Aosaki T, Flaherty AW, Kimura M (1994) The basal ganglia and adaptive motor control. Science 265:1826-1831.

Hilário MR, Clouse E, Yin HH, Costa RM (2007) Endocannabinoid signaling is critical for habit formation. Front Integr Neurosci 1:6.

Hohmann AG, Herkenham M (2000) Localization of cannabinoid CB(1) receptor mRNA in neuronal subpopulations of rat striatum: a doublelabel in situ hybridization study. Synapse 37:71-80.

Kreitzer AC, Carter AG, Regehr WG (2002) Inhibition of interneuron firing extends the spread of endocannabinoid signaling in the cerebellum. Neuron 34:787-796.

Lovinger DM (2007) Endocannabinoid liberation from neurons in transsynaptic signaling. J Mol Neurosci 33:87-93.
Narushima M, Uchigashima M, Hashimoto K, Watanabe M, Kano M (2006) Depolarization-induced suppression of inhibition mediated by endocannabinoids at synapses from fast-spiking interneurons to medium spiny neurons in the striatum. Eur J Neurosci 24:2246-2252.

Partridge JG, Tang KC, Lovinger DM (2000) Regional and postnatal heterogeneity of activity-dependent long-term changes in synaptic efficacy in the dorsal striatum. J Neurophysiol 84:1422-1429.

Ronesi J, Gerdeman GL, Lovinger DM (2004) Disruption of endocannabinoid release and striatal long-term depression by postsynaptic blockade of endocannabinoid membrane transport. J Neurosci 24:1673-1679.

Singla S, Kreitzer AC, Malenka RC (2007) Mechanisms for synapse specificity during striatal long-term depression. J Neurosci 27:5260-5264.

Sjöström PJ, Turrigiano GG, Nelson SB (2003) Neocortical LTD via coincident activation of presynaptic NMDA and cannabinoid receptors. Neuron 39:641-654.

Tepper JM, Abercrombie ED, Bolam JP (2007) Basal ganglia macrocircuits. Prog Brain Res 160:3-7.

Uchigashima M, Narushima M, Fukaya M, Katona I, Kano M, Watanabe M (2007) Subcellular arrangement of molecules for 2-arachidonoylglycerol-mediated retrograde signaling and its physiological contribution to synaptic modulation in the striatum. J Neurosci 27:3663-3676.

Wallmichrath I, Szabo B (2002) Cannabinoids inhibit striatonigral GABAergic neurotransmission in the mouse. Neuroscience 113:671-682.

Wilson CJ (2007) GABAergic inhibition in the neostriatum. Prog Brain Res 160:91-110.

Yin HH, Davis MI, Ronesi JA, Lovinger DM (2006) The role of protein synthesis in striatal long-term depression. J Neurosci 26:11811-11820.

Yin HH, Park BS, Adermark L, Lovinger DM (2007) Ethanol reverses the direction of long-term synaptic plasticity in the dorsomedial striatum. Eur J Neurosci 25:3226-3232. 